\title{
Effect of feedstock properties on extrusion of high aspect ratio micro bi-lumen tubes
}

\author{
Sandeep Kuriakose ${ }^{1^{*}}$, Massimiliano Annoni ${ }^{1}$ \\ ${ }^{1}$ Politecnico di Milano, Department of Mechanical Engineering, 20156, Milan, Italy
}

\begin{abstract}
The micro extrusion of feedstock is a promising emerging technology for manufacturing very high length to thickness aspect ratio metallic micro components which are not feasible by conventional metal processing methodologies or commonly used feedstock processing technologies. Extrusion of high aspect ratio micro components using metallic feedstock confronts the challenges of achieving a continuous extrusion without any breakage, the geometrical accuracy, surface finish and structural properties for the component which are required for the micro application, during micro extrusion process. The type of metallic powder, powder size, type of binder and binder properties are very decisive in making the extrusion process feasible for the micro application. The influence of feedstock properties on micro extrusion of high aspect ratio micro components are still unknown in case of micro extrusion of feedstock. In this research work, the effect of type of feedstock on micro extrusion is studied by extruding micro bi-lumen tubes using bio-compactible steel feedstocks AISI316L and 17-4PH with two different ageing. The geometrical features of the extruded bi-lumen tubes, surface roughness and structural properties are analysed using 3D focus variation microscopy, differential scanning calorimetry (DSC) and scanning electron microscopy (SEM). The analysis showed that feedstock type affects the feasibility of extrusion and geometrical size in great extent. An average Sa roughness deviation from $1.73 \mu \mathrm{m}$ to $4.57 \mu \mathrm{m}$ was observed for feedstocks $17-4 \mathrm{PH}$ and AISI316L. The study also confirms that binder properties and ageing of the feedstocks also have to be taken into account for maintaining the surface finish and structural properties in case of metallic feedstock extrusion of high aspect ratio bi-lumen tubes.
\end{abstract}

Keywords: micro extrusion, bi-lumen tube, feedstock properties, surface quality, powder metallurgy

\section{Introduction}

Feedstock processing technologies are widely used in the recent decades for the manufacturing of micro components of unique properties by the use of powder metallurgical principle. Feedstock, which is a compound made of metal and polymeric binders, is formed using the forming technologies like injection moulding, hot pressing, extrusion etc. [1, 2]. This formed part in a state called green-state is further processed by debinding and sintering to remove the polymeric content and to obtain the strength respectively [3]. The generation of high aspect ratio (length/thickness) metallic components is still an unattainable target by mostly used feedstock processing methodologies such as injection moulding and hot pressing because of the problem in size of the die that can be used, part ejection, non-uniformity in the feedstock flow, binder separations etc. [1].

The micro extrusion is the only processing methodology that can be used for manufacturing of very high aspect ratio components particularly for several bio-medical and micro fluidics applications [4]. The micro extrusion of ceramics and hard metals is used by the companies for the commercial productions but micro extrusion of metallic feedstock is still in development stage. The powder size and suitable flow properties of feedstock are very important for the continuous extrusion of micro features $[1,3,5]$. The limitations in available metallic feedstock with powder sizes in nano metric size due to the difficulty in powder manufacturing, the lack of knowledge regarding the suitable binder material and metal-binder ratios appropriate for extrusion, limit the advancements in metallic feedstock extrusion $[1,3,5]$. The feedstock properties also could change with respect to the ageing by the changes happening to the binder polymer [6]. A study regarding the effect of ageing of feedstock on extrusion properties also is lacking. In this study, the influence of type of feedstock and effect of ageing of feedstock on micro extrusion of high aspect ratio micro components is studied by extruding micro bi-lumen tubes of nominal outer diameter $3.18 \mathrm{~mm}$, lumen diameters $0.7 \mathrm{~mm}$ and length $400 \mathrm{~mm}$ using three biocompactible steel feedstocks at different states AISI316L, 17-4PH and 17-4PH aged. The extruded bilumen tubes surface roughness and structural properties are evaluated using 3D focus variation microscopy, differential scanning calorimetry (DSC) and scanning electron microscopy (SEM). Extruded tubes in green-state are further debinded, sintered and final property variations are analysed.

\section{Materials and method}

\subsection{Materials}

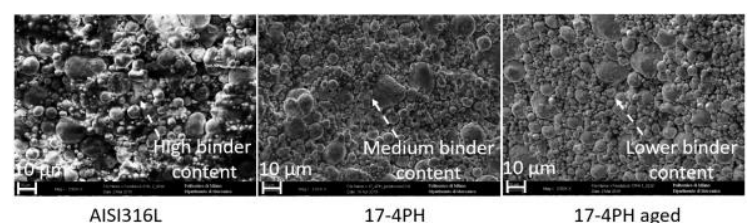

Fig. 1. SEM image of feedstock, AISI316L D110E, 17-4PH G120E and 17-4PH G120E aged.

The feedstock materials used for experimental investigation are commercial feedstocks, AISI316L D110E, 17-4PH G120E and 17-4PH G120E aged (1.5 years) of Polymim (Polymim Gmbh, Germany). The feedstock material in the form of pellets of size 2-4 mm are used for the extrusion experiments. The processing temperature of all the feedstocks were assessed using DSC. The DSC analysis showed a processing temperature ranges of $140-200{ }^{\circ} \mathrm{C}$ for all the three feedstocks. The SEM (see Fig.1) and electric discharge spectroscopy (EDS) analysis of feedstock verified the homogeneity of feedstock components and the constituting elements of the biocompatible steels in 


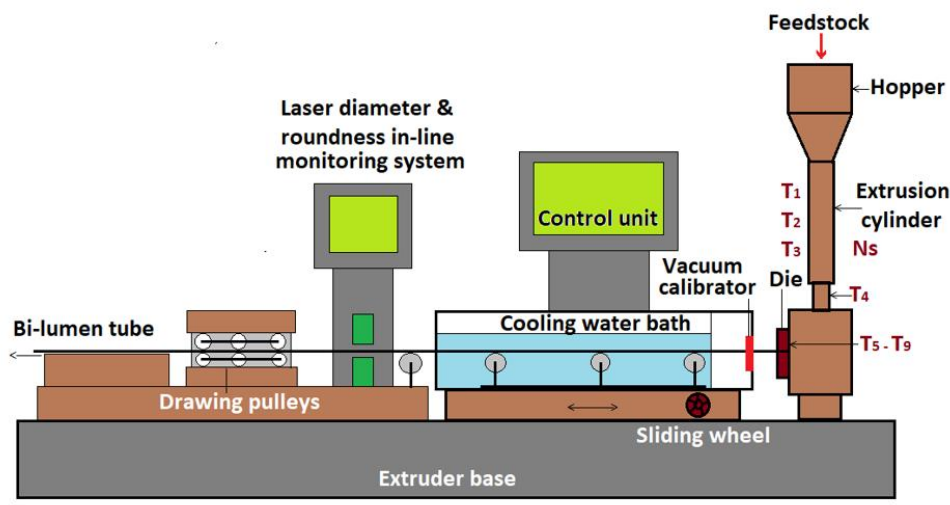

Fig. 2. The schematic diagram of the micro extruder showing the process sequence of micro extrusion

both compositions. Steel particles were observed of similar shapes and size in all the three feedstocks with most of the particle diameters below $10 \mu \mathrm{m}$ and with particle size varying from 1 to $30 \mu \mathrm{m}$ by SEM examination. The binder percentage in AISI316L $\mathrm{D} 110 \mathrm{E}$ is found to be higher than in the other two. The binder of all the feedstock compositions are waterdebindable and then thermal-debindable.

\subsection{Experimentation}

A single screw extruder (Gimac S.r.l. Italy) typically used for the polymer extrusion with a $12 \mathrm{~mm}$ extrusion screw diameter, available in company Enki s.r.l. is used for the feedstock micro extrusion study. The schematic diagram of the system with associated equipment is shown in Fig. 2. The extruder is designed with four heaters $\left(T_{1}-T_{4}\right)$ on extrusion cylinder and five heaters $\left(T_{5}-T_{9}\right)$ on the die in such a way to keep the heat distribution uniform. The process parameters in case of extrusion using screw extruder are temperature of extrusion ( $\left.\mathrm{Te}=\mathrm{T}_{1}-\mathrm{T}_{9}\right)$, screw speed $(\mathrm{Ns})$, vacuum in the vacuum calibrator, temperature of the water bath and the rotation speed of drawing pulley. The piolet experiments showed that unlike the polymer extrusion, the only influencing parameters in case of feedstock extrusion which determine the properties of the extruded bi-lumen tubes are $\mathrm{Te}$ and Ns. The die and pin used for bi-lumen tube extrusion had a nominal diameter of $3.18 \mathrm{~mm}$ for the tube and $0.70 \mathrm{~mm}$ for each lumen. The feedstock put into the hopper is pushed between pin and die by a nonstop rotation of the screw and the bi-lumen cross section is extruded continuously.

Table 1. Extrusion parameters used for bi-lumen tube extrusion

\begin{tabular}{lccc}
\hline Feedstock materials & $\begin{array}{c}\mathrm{Te} \\
{\left[{ }^{\circ} \mathrm{C}\right]}\end{array}$ & $\begin{array}{c}\mathrm{Ns} \\
{[\mathrm{rpm}]}\end{array}$ & $\begin{array}{c}\text { Replicat } \\
\text { ions }\end{array}$ \\
\hline $\begin{array}{l}\text { AISI316L D110E } \\
\text { 17-4PH G120E }\end{array}$ & 160 & 24 & 3 \\
$\begin{array}{l}\text { 17-4PH G120E (1.5 years } \\
\text { aged) }\end{array}$ & & & \\
\hline
\end{tabular}

A total of 9 experiments are conducted by extruding high aspect ratio $(\mathrm{L} / \mathrm{Dt}=125 ; \mathrm{L} / \mathrm{DI}=570)$ micro bi-lumen tube samples of nominal tube diameter $\mathrm{Dt}=3.18 \mathrm{~mm}$, lumen diameter $\mathrm{DI}=0.7$ and length $\mathrm{L}=$ $400 \mathrm{~mm}$ by using 3 different feedstock materials for 3 replications. The used extrusion parameters are shown in Table 1. The extruded bi-lumen tubes in green-state are showed in Fig. 3. The extruded tubes in green-state are analysed for surface roughness and structural property changes.

The extruded samples are further debinded, presintered and sintered to understand the variation of the surface and structural properties. The bi-lumen tubes extruded using feedstock AISI316L were of very poor surface finish and were eliminated from further processing.

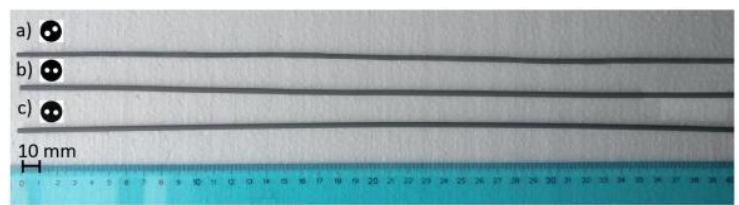

Fig. 3. Micro bi-lumen tubes (length $=400 \mathrm{~mm}$ ) extruded in green-state using a) AISI316L D110E, b) 17-4PH G120E aged and c) 17-4PH G120E (inset: magnified cross-section).

The samples of $17-4 \mathrm{PH}$ and $17-4 \mathrm{PH}$ aged were further debinded, pre-sintered and sintered by cutting a length of $125 \mathrm{~mm}$ from each sample of $400 \mathrm{~mm}$. Water debinding is done in a debinding set up using distilled water and $2 \%$ INHIBITOR 4000 (Zschimmer \& Schwarz, Germany) corrosion inhibitor with a debinding temperature of $50^{\circ} \mathrm{C}$ for a debinding time of $72 \mathrm{hrs}$ [7]. Thermal debinding and pre-sintering operations are done one after the other by heating the sample to the thermal debinding temperature of $600^{\circ} \mathrm{C}$ at a heating rate of $3{ }^{\circ} \mathrm{C} / \mathrm{min}$ and holding the samples for $2.5 \mathrm{hrs}$ and then heating at a higher heating rate of $4{ }^{\circ} \mathrm{C} / \mathrm{min}$ to the pre-sintering temperature of $800^{\circ} \mathrm{C}$ for a holding time of $1 \mathrm{hr}$ followed by natural slow cooling [7, 8]. A Graphite tubular furnace on Alumina plates with $\mathrm{H}_{2}$ atmosphere is used for thermal debinding and presintering cycle. The $100 \% \mathrm{H}_{2}$ atmosphere is selected for the pre-sintering and sintering activity as $\mathrm{H}_{2}$ or Vacuum were found to be least corrosive for the parts [9]. A small heating rate was selected for the debinding process to avoid the surface defects considering the micro lumens and high aspect ratio of the bi-lumen tubes. Thermal debinding and sintering were decided to be done in separate furnaces to minimize the possible contamination and corrosion which will prevent the biomedical use for which the tubes may find applications in the future. The sintering of one sample of $17-4 \mathrm{PH}$ and $17-4 \mathrm{PH}$ aged are done in a vertical tubular furnace (TAV S.p.a., Italy) by heating the sample to $1380{ }^{\circ} \mathrm{C}$ at a heating rate of $5{ }^{\circ} \mathrm{C} / \mathrm{min}$ and holding the samples for $2 \mathrm{hr}$ and cooling down naturally [8]. 
a)

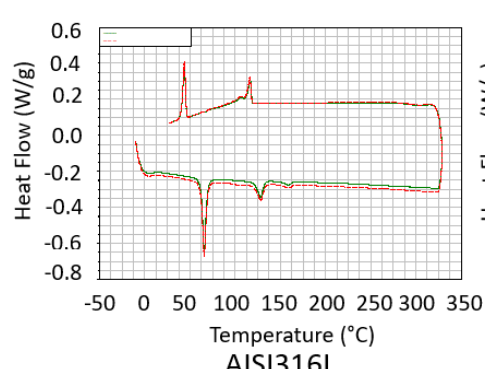

b)

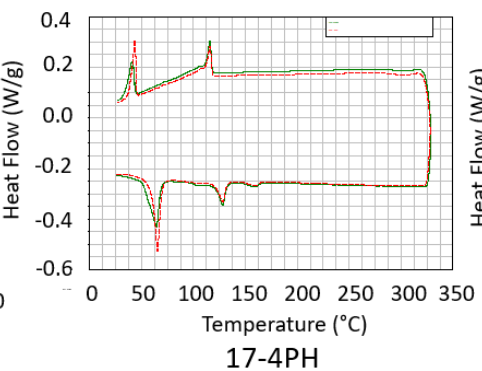

c)

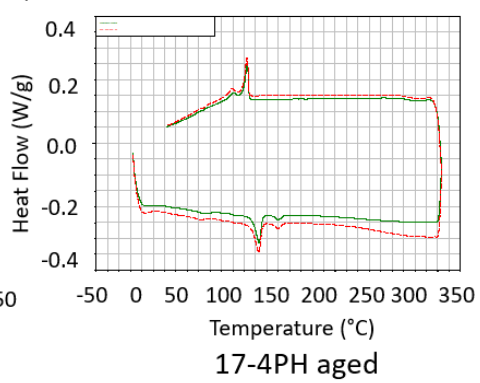

a)

Fig. 4. DSC of the feedstock pellets a) AISI316L b) $17-4 \mathrm{PH}$, and c) $17-4 \mathrm{PH}$ aged

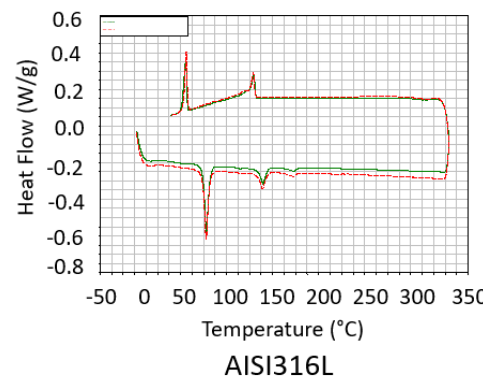

b)

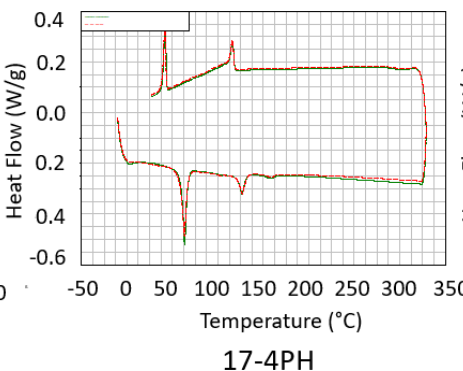

c)

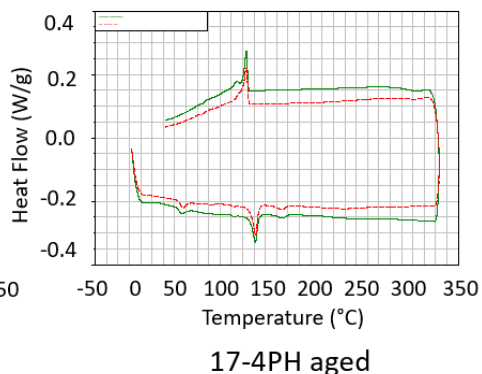

Fig. 5. DSC analysis of the extruded tubes of a) AISI316L b) $17-4 \mathrm{PH}$, and c) $17-4 \mathrm{PH}$ aged.

The bi-lumen samples at green-state and sintered state were analysed to understand surface and structural properties. The areal surface roughness (Sa) of the tubes were evaluated by acquiring a surface of $1000 \times 1000 \mu \mathrm{m}^{2}$ at 2 points along the length direction and 2 points around circumferential direction. This approach was adopted to understand the roughness variations along length and circumferential directions. A focus variation optical microscope (Alicona, Austria) with a 20X lens and with a vertical resolution of 0.08 $\mu \mathrm{m}$ and a horizontal resolution of $2.5 \mu \mathrm{m}$ were used for the acquisition. The form removal of the acquired data clouds of the surface are carried out using a cylindrical function and Sa was calculated by using a filter of 150 $\mu \mathrm{m}(15 \%$ of the $1000 \mu \mathrm{m})$. The structural property variations of bi-lumen tubes such as steel particle attachments, carbon content, porosity etc. are analysed using SEM (Zeiss, Germany) with an associated EDS (Oxford instruments, UK).

\section{Results and discussion}

The feasibility of extrusion of bi-lumen tubes using feedstock and extruded tubes' properties very much depend on the type of feedstock and the feedstock properties. The micro extrusion with AISI316L feedstock took a long time to get a steady state condition and the extruded tubes in green-state were found to be very fragile and with poor surface quality. The extrusion study with $17-4 \mathrm{PH}$ and $17-4 \mathrm{PH}$ aged also differed in the time needed to achieve the steady state for extrusion. 17-4PH took much less time compared to $17-4 \mathrm{PH}$ aged feedstock. The study of the geometrical reproduction, surface quality and structural properties gave the following results.

\subsection{DSC analysis of samples}

The DSC analysis of the feedstock pellets and extruded bi-lumen tubes were carried out to understand the thermal properties of the feedstock material used for extrusion and also to understand the variations happening to them by extrusion.
DSC analysis of the feedstock pellets of AISI316 L and 17-4PH showed similar behavior with same melting (negative) peak and crystallization (positive) peaks for the binder components at $50-60^{\circ} \mathrm{C}$ and 130 $140^{\circ} \mathrm{C}$ in the same heat flow intervals (see Fig. $4 \mathrm{a}$ and b). This suggests that the polymeric binder with similar properties were used in both feedstocks. The analysis of the 17-4PH aged showed a loss of major part of the components of the binder which is having a melting temperature near $60{ }^{\circ} \mathrm{C}$ (see Fig. $4 \mathrm{~b}$ and $\mathrm{c}$ ). These components could be wax or other surfactants which have melting point of that range and are mostly removed by the water debinding process.

The DSC analysis of the samples from extruded tubes were carried out to understand the property changes happening to the feedstock material of the tubes by extrusion and subsequent cooling step by passing the hot extruded tubes through cooling water. The DSC analysis of the extruded tubes showed similar behavior that of the pellets of the feedstock. The same trends were observed for all the three feedstocks (see Fig. $5 \mathrm{a}, \mathrm{b}$ and $\mathrm{c}$ ). This behavior of the DSC curves states that there is no significant variation of the properties of the binder components by extrusion.

3.2 Bi-lumen tubes' surface quality and structural analysis

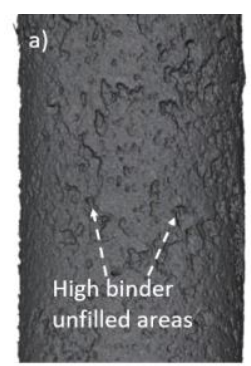

AISI316L

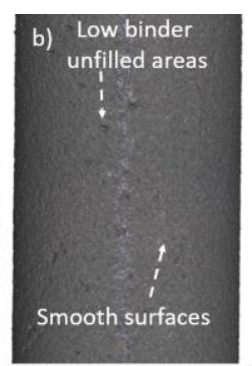

17-4PH

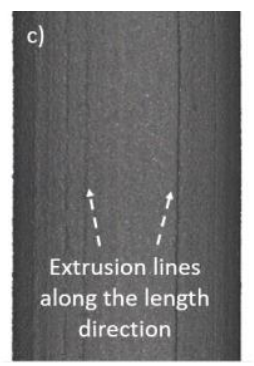

17-4PH aged
Fig. 6. 3D microscope images of the surfaces of tubes extruded using a) AISI316L, b) 17-4PH and c) $17-4 \mathrm{PH}$ aged. 
The surface roughness, Sa analysis showed high variability between different tubes as shown in Fig. 6. A highest average Sa green-state value of $4.57 \mu \mathrm{m}$ for AISI316L feedstock, followed by a Sa of $2.05 \mu \mathrm{m}$ for $17-4 \mathrm{PH}$ aged and $1.73 \mu \mathrm{m}$ for $17-4 \mathrm{PH}$ were observed. The variability of the green-state Sa was found to be the highest for the AISI316L and the least for $17-4 \mathrm{PH}$ (see Fig. 7). Sa analysis of 17-4PH aged also showed an increase in average roughness value and variability by ageing of the feedstock.

The 3D microscopic images of the surfaces of tubes extruded using AISI316L showed random crests and troughs with feedstock agglomeration and binder unfilled areas (see Fig. 6 a). The surfaces of the bilumen tubes extruded with 17-4PH generally showed good smooth surface without any feedstock agglomeration crests or troughs (see Fig. 6 b). Rarely in some areas, minor binder unfilled spaces between the particles were observed. The surfaces of the bilumen tubes extruded using 17-4PH aged generated extrusion lines along the length direction of the tubes (see Fig. $6 \mathrm{c}$ ). The surface and structural analysis by SEM provide clearly the reasons for these particular changes.

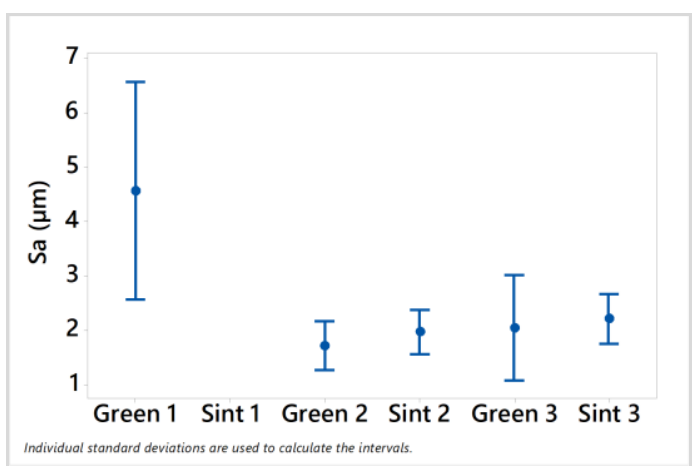

Fig. 7. Interval plot for Sa values of extruded bi-lumen tubes at green and sintered states of 1) AISI316L 2) $17-4 \mathrm{PH}$ and 3) $17-4 \mathrm{PH}$ aged.

The SEM analysis of pellets of the feedstock AISI316L showed a higher binder content compared to $17-4 \mathrm{PH}$ and $17-4 \mathrm{PH}$ aged. The very high binder content of the feedstock increased the fluidity and the extrusion resulted in agglomerations with crests and troughs along the surface of the tubes (see Fig. 8).

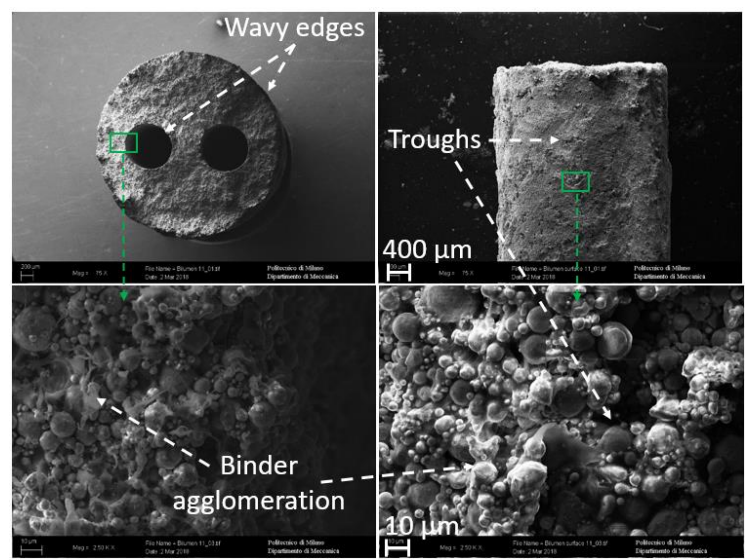

Fig. 8. SEM image analysis of bi-lumen tubes extruded using AISI316L

In addition to the binder content, the surface properties of AISI316L compared to 17-4PH also could be a reason for this particular behavior and the nonuniform flow resulted because of this created wavy boundaries for the tube diameters and inner lumens. All these effects resulted in very high Sa values for the bi-lumen tubes extruded using AISI316L.

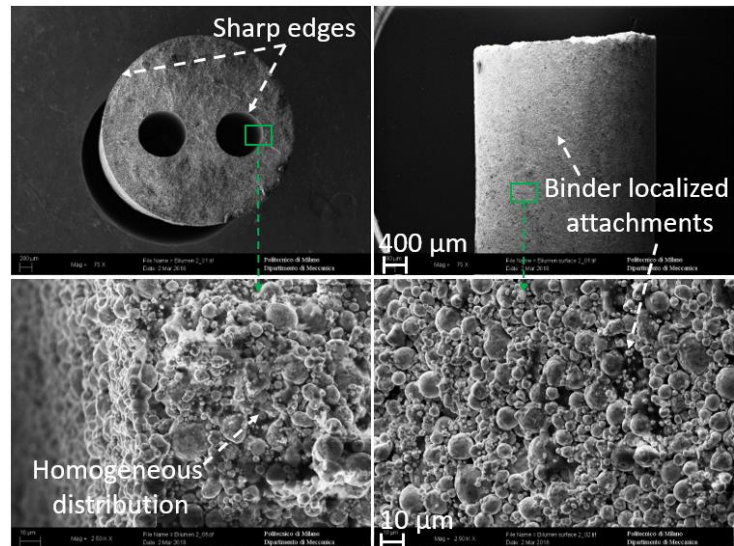

Fig. 9. SEM image of bi-lumen tube extruded using 17-4PH

The SEM of the cross-section of the tubes extruded with 17-4PH showed sharp edges for the tubes and lumens and rarely any porosity (see Fig. 9). The SEM of the surface of the tubes extruded with 17-4 $\mathrm{PH}$ generally produced a good surface without any agglomerations and hence with the least Sa. Some minor binder localized extra attachments were observed on the surface of the bi-lumen tubes. These localized attachments do not create any surface deviations on the extruded bi-lumen tubes. This could be probably produced by the separated binders because of extrusion pressure and sticking to the extrusion die and come attached on the surface of the bi-lumen tubes during continuous extrusion. The good surface and structural properties showed that the binder percentage and flow properties of the feedstock are correct for the $17-4 \mathrm{PH}$.
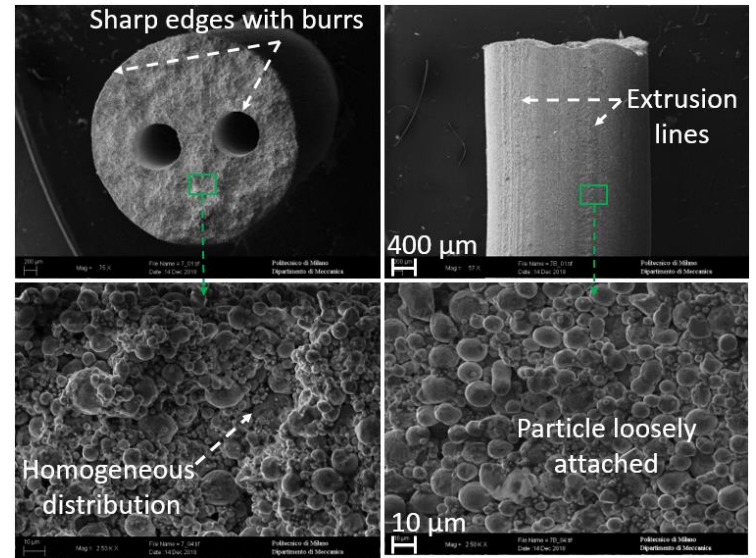

Fig. 10. SEM image of bi-lumen tube extruded using 17-4PH aged

The ageing of the 17-4PH feedstock resulted in losing of some components of the binder. This reduction in binder components generated extrusion lines along the extrusion direction of the bi-lumen tube surface as seen in the SEM Image (see Fig. 10). This extrusion line formation is caused by detachment of some metal particles from the feedstock during extrusion and sticking to the die of the extruder. This 
can be confirmed from the loosely attached metal particle by binders in magnified SEM image of the surface where lines were observed (see Fig. 10). This caused an increase of Sa values for 17-4 PH aged tubes compared to 17-4PH tubes. The SEM of the cross-sections showed generally sharp edges for the tubes extruded with 17-4PH aged feedstock but these extrusion lines result in minor burr like attachments to the boundaries of the tubes. The SEM cross-section analysis for the 17-4PH aged feedstock showed homogeneous feedstock distribution and similar porosities compared to the feedstock without ageing for the experimented extrusion condition. This states that flow properties of the aged feedstock were still good for the extrusion of bi-lumen tubes in the selected extrusion condition.

\subsubsection{Properties at the sintered state}

The surfaces of sintered tubes exhibited a shining surface with good surface quality without any cracks or defects by the sintering operation. The sintering of the tubes extruded using 17-4PH and 17-4PH aged showed a slight increase in the Sa value proportional to the $\mathrm{Sa}$ in the corresponding green-state (see Fig. 7).

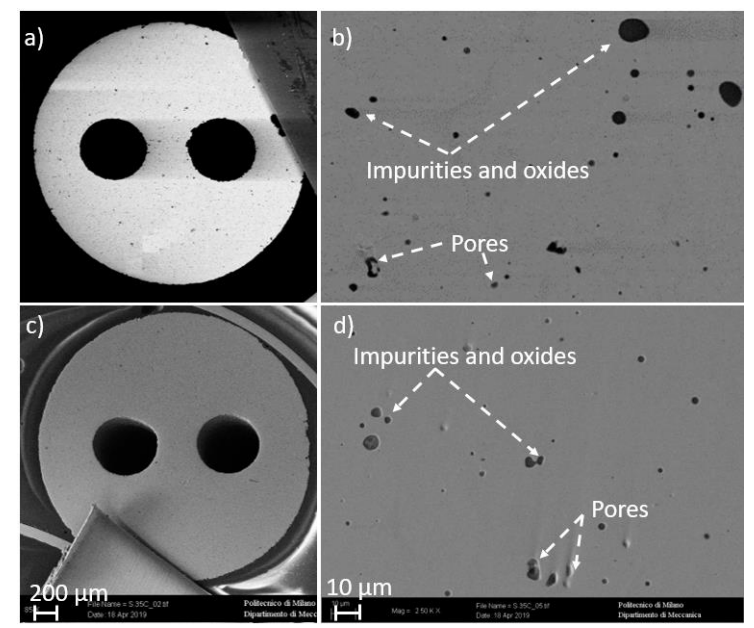

Fig 11. SEM Image of cross-section of bi-lumen tube at sintered state of; $a$ \& b) $17-4 \mathrm{PH}$ and c \& d) 17-4PH aged.

The interval plot also showed that variability in Sa is reduced by sintering $17-4 \mathrm{PH}$ aged. This means that higher irregularities observed with 17-4PH aged were reduced a little by detachment of loosely attached particle during debinding and bond formation between the metal particles by sintering. The sintering eliminated the porosity to a great extent but defects and extrusion marks produced during extrusion were still retained on the surface even though in a diminished manner. The SEM image of the crosssection of the bi-lumen tubes of 17-4PH (see Fig. 11 a $\&$ b) and 17-4PH aged (see Fig. $11 \mathrm{c} \& \mathrm{~d}$ ) showed similar properties. Some pores of the size of 1-6 $\mu \mathrm{m}$ in addition to some dark small globules were detected in the cross sections of the tubes. The EDS, analysis showed that these globules comprise mainly Silicon and Oxygen. The size of these globules was found to be slightly higher for $17-4 \mathrm{PH}$ than $17-4 \mathrm{PH}$ aged and this suggests that these globules could probably due to contamination by foreign particles attached with binders of the feedstock pellets and from the alloying elements in the steel.

\section{Conclusions}

The extrusion study to understand the effect of feedstock properties on extrusion behaviour in case of extrusion of high aspect ratio micro bi-lumen tubes showed that feedstocks and their binder contents influence the surface and structural properties of the extruded tubes very much. A variation in average roughness value of Sa from $1.73 \mu \mathrm{m}$ to $4.57 \mu \mathrm{m}$ was observed for a change of feedstock material from 17$4 \mathrm{PH}$ to AISI316L and a corresponding change of type of metal particle and the binder content. The study also showed that ageing of the feedstock could affect the extrusion behaviour by affecting the surface properties specially than the structural properties. The sintering of the bi-lumen tubes produced by extrusion showed a good structural integrity with minimal pores but retained the surface irregularities produced during extrusion in diminished way. This suggest that selecting the right feedstock with suitable binder quantity is essential in controlling the surface and structural properties in extrusion of high aspect ratio micro lumen tubes.

\section{Acknowledgements}

This research work was undertaken in the context of MICROMAN project ("Process Fingerprint for Zerodefect Net-shape MICROMANufacturing", http://www.microman.mek.dtu.dk/). MICROMAN is a European Training Network supported by Horizon 2020, the EU Framework Programme for Research and Innovation (Project ID: 674801). Thanks go also to Mr. Sanna (Enki s.r.l.), Eng. Cataldo and Dr. Parenti (Politecnico di Milano), Dr. De Gaudenzi and Eng. Garabelli (Films S.p.a.), Eng. Fiorese and Mr. Gionda (TAV S.p.a.) for their support.

\section{References}

[1] U.M. Attia, et al., "A review of micro-powder injection moulding as a microfabrication technique," J. Micromechanics Microengineering., 2011; 21: 1-22.

[2] S.B. Li, et al., "Fabrication of thin-walled 316L stainless steel seamless pipes by extrusion technology", J. Mater. Process. Technol., 2007; 183: 57-61.

[3] G. Joamin, et al., "Additive manufacturing of metallic and ceramic components by the material extrusion of highly-filled polymers: A Review and Future Perspectives, "Materials., 2018; 11:840.

[4] C. Rauwendaal, Polymer Extrusion, Hanser Publishers, Munich 2014 ISBN 9781569905166.

[5] B. Hausnerova, et al., "Rheological properties of gas and water atomized 17-4PH stainless steel MIM feedstocks: Effect of powder shape and size," Powder Technol., 2017; 312:152-158.

[6] H. F Giles, et. al., "Extrusion: The Definitive Processing Guide"; William Andrew, New York 2005 ISBN 0815514735.

[7] M.T. Zaky, "Effect of solvent debinding variables on the shape maintenance of green molded bodies," J. Mater. Sci., 2004; 39: 3397-3402.

[8] Y. Wu, et al., "Effects of residual carbon content on sintering shrinkage, microstructure and mechanical properties of injection molded 17-4 $\mathrm{PH}$ stainless steel," J. Mater. Sci., 2002; 37: 3573- 3583. [9] R.M. German, "MIM 17-4 PH Stainless Steel: Processing, properties and best practice", Powder Injection Moulding International. June 2018. 12: 2, 4976. 